\title{
ESTUDIO DE VALIDEZ Y CONFIABILIDAD DEL CUESTIONARIO NÓRDICO ESTANDARIZADO, PARA DETECCIÓN DE SINTOMAS MUSCULOESQUELÉTICOS EN POBLACIÓN MEXICANA
}

\author{
VALIDITY AND RELIABILITY STUDY OF THE STANDARDIZED NORDIC \\ QUESTIONNAIRE, FOR THE DETECTION OF MUSCULAR-SKELETAL SYMPTOMS IN \\ MEXICAN POPULATION
}

Elvia Luz González Muñoz*

\begin{abstract}
Resumen: Con el objetivo de comprobar la confiabilidad y validez del cuestionario nórdico original, traducido al español, se realizó este estudio con trabajadores de la zona occidente de México. Se pretende contar con un instrumento útil tanto para investigación como para el tamizaje en el sector productivo, ya que es un instrumento de fácil y rápida aplicación. Se realizó un estudio de tipo transversal, descriptivo, con una muestra a conveniencia de 585 trabajadores mexicanos, tanto operativos como administrativos provenientes de seis empresas del área metropolitana de Guadalajara. El análisis de confiabilidad arrojó un alfa de Cronbach de o,863, considerado muy bueno. Posteriormente, se realizó un análisis factorial exploratorio para comprobar la estructura del instrumento; los resultados indican un valor de bondad de ajuste de $\chi^{2}=550, p \leq 0,001$. Se obtuvo una medida de adecuación muestral con un valor $\mathrm{KMO}=0,822$, el cual indica un buen ajuste. A partir de la extracción de factores se obtienen nueve, correspondientes a los segmentos corporales analizados, los cuales explican el 72,05\% de la varianza total. Los resultados obtenidos confirman la confiabilidad y validez del instrumento, y presentan valores similares a los reportados en otros estudios. Se analizó la validez mediante una prueba test - retest, manteniéndose los resultados. Una de las ventajas de utilizar el instrumento original es que permite la comparación, entre diversos estudios, no así el resto de los instrumentos a los cuales se les han realizado diversas modificaciones y que en ocasiones no son reportadas con claridad.
\end{abstract}

Palabras clave: Molestias musculoesqueléticas, validación, trabajadores mexicanos, cuestionario nórdico.

\begin{abstract}
In order to verify the reliability and validity of the original nordic questionnaire, translated into Spanish, this study was carried out with workers from the western part of Mexico. It is intended to have a useful instrument both for research and for screening in the productive sector, since it is an instrument that is easy and quick to apply. A cross-sectional, descriptive study was carried out with a convenience sample of 585 Mexican workers, both operational and administrative, from 6 companies in the metropolitan area of Guadalajara. The reliability analysis yielded a Cronbach's alpha of 0.863 , considered particularly good. An exploratory factor analysis was subsequently carried out to verify the structure of the instrument, the results indicate a goodness-of-fit value of $\chi^{2}=550, p \leq 0.001$. A sample adequacy measure was obtained with a $\mathrm{KMO}$ value $=0.822$, which indicates a good fit. From
\end{abstract} $\begin{array}{lcccc}\text { *Universidad de Guadalajara. } & \text { Guadalajara, México. Correo } & \text { electrónico: } \\ \text { elvia.gmunoz@academicos.udg.mx. Orcid: https://orcid.org/oooo-ooo3-0353-1723 } & \end{array}$ 
the factor extraction, nine factors are obtained, corresponding to the analyzed body segments, which explain $72.05 \%$ of the total variance. The results obtained confirm the reliability and validity of the instrument, and present values similar to those reported in other studies. Validity was analyzed by means of a test-retest, maintaining the results - One of the advantages of using the original instrument is that it allows comparison between different studies, but not the rest of the instruments to which various modifications have been made. and that are sometimes not clearly reported.

Keywords: Musculoskeletal discomfort, validation, mexican workers, nordic questionnaire.

Recepción: 25.04.2021 / Revisión: 30.04.2021 / Aceptación: 07.05.2021

\section{Introducción}

A partir del año 2020, en México entró en vigor una nueva norma encaminada a la identificación, análisis, prevención y control de factores de riesgo ergonómico (NOM-036-1STPS de 2018). Como parte de la norma se recomienda el uso del cuestionario nórdico, con el fin de detectar la presencia de dolor y síntomas musculoesqueléticos. Esto generó la necesidad de contar con un instrumento validado para emplearse en los centros de trabajo. Existe la necesidad de adoptar medidas preventivas y acciones para la detección temprana de trabajadores que pudieran presentar síntomas o molestias musculoesqueléticas, que a la larga se traducirán en lesiones. La falta de esta detección oportuna implica riesgo para los trabajadores, así como fuertes gastos para las empresas tanto en el pago de incapacidades, aumento de la prima de riesgo, así como los derivados del ausentismo y de la baja productividad de los trabajadores.

De acuerdo al reporte generado por el Instituto Mexicano del Seguro Social (2021), entre el año 2015 y 2019, ha aumentado el porcentaje de enfermedades de trabajo en cuyo origen se encuentran riesgos ergonómicos (figura 1), así como el porcentaje de incapacidades permanentes, principalmente en mujeres, como se presenta en la figura 2. 
Figura 1. Enfermedades de trabajo relacionadas con riesgos ergonómicos en los años 2015-2019.

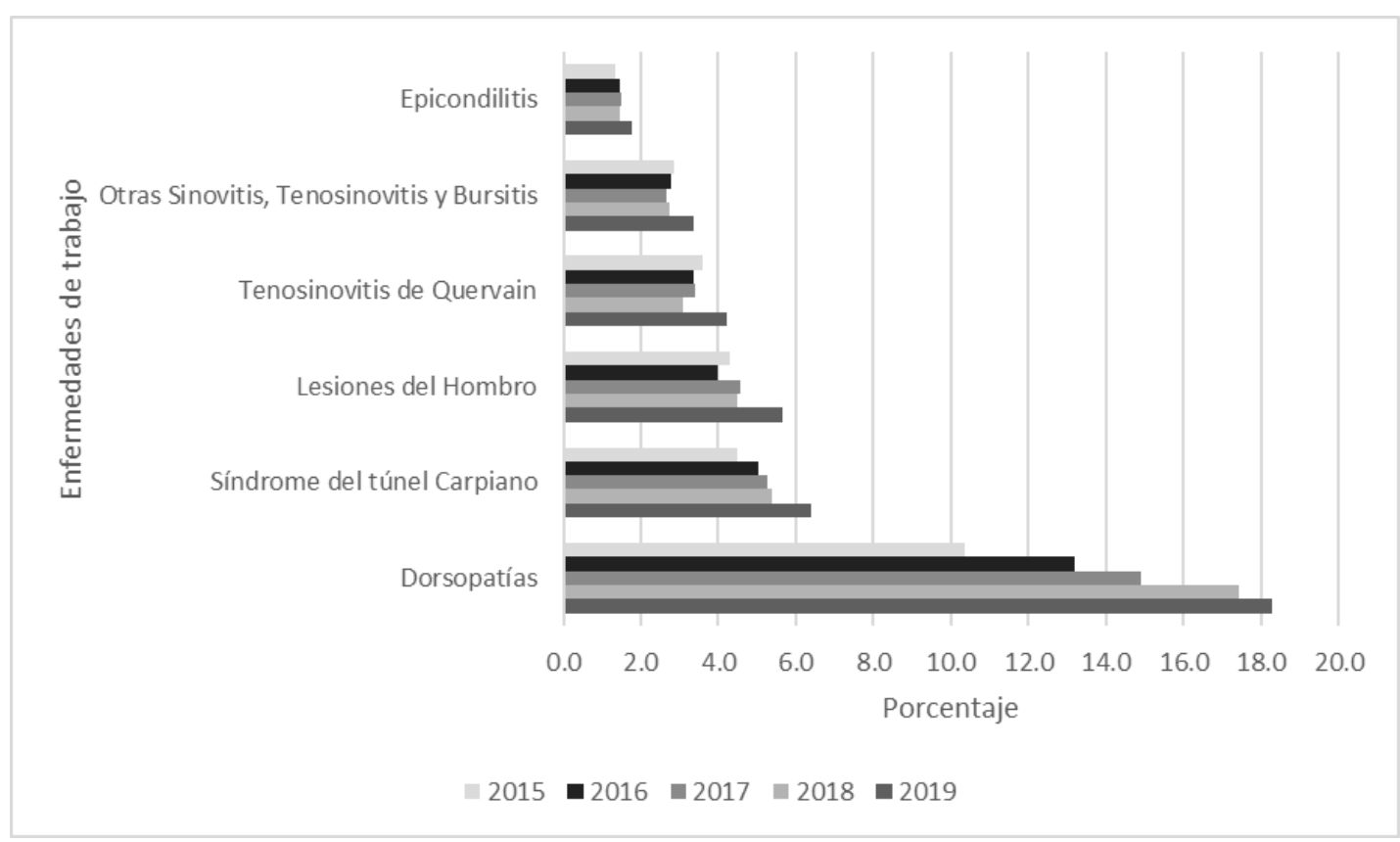

Figura 2. Incapacidades permanentes relacionadas con riesgos ergonómicos en el año 2019.

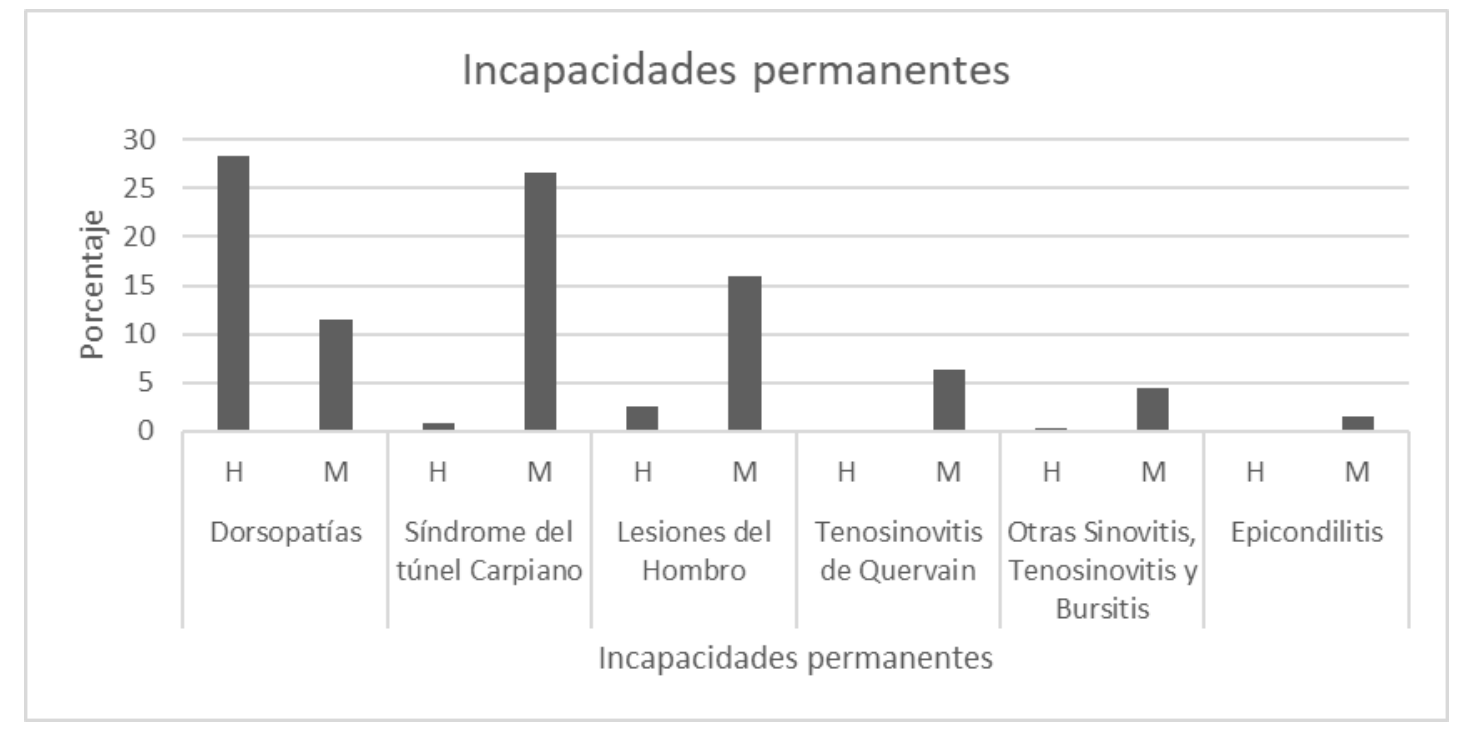

Un tamizaje de los trabajadores, con instrumentos sencillos, en conjunto con la realización de evaluaciones ergonómicas de los puestos de trabajo, permitirán realizar acciones correctivas para mejorar las condiciones de trabajo.

Uno de los instrumentos más reconocidos es el Cuestionario Nórdico Estandarizado, el cual fue elaborado con la finalidad de tener un instrumento estandarizado que permita registrar y analizar los síntomas musculoesqueléticos, a fin de compararlos entre diversos estudios. Asimismo, es un instrumento de evaluación inicial en los lugares de trabajo. Su estructura original es un cuestionario binario y de elección múltiple dividido en dos apartados, uno general para la evaluación de nueve segmentos corporales y otro específico enfocado en la espalda baja y los hombros. En su validación reportó la evaluación de la 
confiabilidad en una prueba - retest con un porcentaje de acuerdo de o a 15\% (Kourinka, et al., 1987).

Se han realizado distintas versiones del instrumento, las cuales han reportado su confiabilidad, sin embargo, para su uso es necesario considerar en donde serán utilizados. En la tabla 1 se presentan algunos de los estudios en los cuales se reportan evaluaciones de la confiabilidad del instrumento, empleando diversas técnicas.

Tabla 1. Estudios de confiabilidad del cuestionario nórdico.

\begin{tabular}{|c|c|c|c|}
\hline Referencia & Población (n) & Método de evaluación & Resultado \\
\hline $\begin{array}{l}\text { Kuorinka et al. } \\
\text { (1987). }\end{array}$ & $\begin{array}{ll}\text { Ingenieros } & \text { (29), } \\
\text { recepcionistas } & \\
\text { médicos (17), y } & 22 \\
\text { trabajadores } & \text { de } \\
\text { mantenimiento. } & \end{array}$ & $\begin{array}{l}\text { Método test - retest. } \\
\text { Validación del test a través } \\
\text { de historias clínicas. }\end{array}$ & $\begin{array}{l}\text { El número de } \\
\text { respuestas } \\
\text { idénticas varia de o a } \\
23 \% \text {. } \\
\text { La variación en las } \\
\text { respuestas es de o a } \\
20 \% \text {. }\end{array}$ \\
\hline $\begin{array}{l}\text { Rojas y Velarde } \\
(2020) .\end{array}$ & $\begin{array}{lr}\text { Trabajadores } & \text { de } \\
\text { vigilancia } & y \\
\text { seguridad }(n=86) . & \end{array}$ & Alfa de Cronbach. & $\alpha=0,8$. \\
\hline $\begin{array}{l}\text { Zamora-Chávez et } \\
\text { al. (2020). }\end{array}$ & $\begin{array}{l}\text { Trabajadores de } \\
\text { limpieza del servicio } \\
\text { de } \\
(\mathrm{n}=129) \text {. }\end{array}$ & $\begin{array}{l}\text { Coeficiente de } \\
\text { Richarson. }\end{array}$ & Rtt $=0,727$ y 0,816 \\
\hline $\begin{array}{l}\text { Maradei et al. } \\
\text { (2019). }\end{array}$ & Agricultores $(n=72)$. & $\begin{array}{l}\text { Confiabilidad test - retest } \\
\text { y alfa de Cronbach. }\end{array}$ & $\begin{array}{lr}\text { Presenta } & \text { una } \\
\text { confiabilidad test } & - \\
\text { retest de } 0,77 \text { y de } & \text { un } \\
\text { o\% a 20\% de } & \text { desacuerdo entre las } \\
\text { respuestas } & \text { del } \\
\text { cuestionario y el } \\
\text { historial clínico de los } \\
\text { participantes. } \\
\text { Alfa de Cronbach de } \\
\text { o,896. }\end{array}$ \\
\hline $\begin{array}{l}\text { Martínez y Muñoz, } \\
\text { (2017). }\end{array}$ & 54 trabajadores. & Test - & $\begin{array}{l}\text { Concordancia entre } \\
0,119 \text { y } 0,361 .\end{array}$ \\
\hline $\begin{array}{l}\text { Bellorín et al. } \\
\text { (2007). }\end{array}$ & $\begin{array}{l}89 \text { trabajadores } \mathrm{de} \\
\text { una empresa de } \\
\text { construcción civil. }\end{array}$ & $\begin{array}{lll}\text { Coeficiente } & \text { de } & \text { Kuder } \\
\text { Richarson. } & & \end{array}$ & $\begin{array}{l}\text { Confiabilidad de }- \text { Rtt } \\
=0,83 .\end{array}$ \\
\hline
\end{tabular}

Una de las ventajas del cuestionario original, es su simplicidad, lo cual es muy importante si se consideran las características de la población a evaluar. Los trabajadores del área de producción en México cuentan en promedio con un nivel educativo medio básico, y los instrumentos que se utilicen para su evaluación deben ser lo más sencillos posibles.

El objetivo del presente artículo es presentar la validación de la versión original, realizada con trabajadores mexicanos, y con ello tener un instrumento útil tanto para investigación como para el tamizaje en el sector productivo, ya que es un instrumento de fácil y rápida aplicación, pero confiable. 


\section{Materiales y métodos}

Se realizó un estudio de tipo transversal, descriptivo, con una muestra a conveniencia de 585 trabajadores mexicanos, tanto operativos como administrativos provenientes de seis empresas del área metropolitana de Guadalajara. Se obtuvo el consentimiento informado de los trabajadores, señalándoles que la participación era libre y si ellos no deseaban participar, podían retirarse en el momento que consideraran conveniente.

\section{Instrumentos}

Se utilizó el cuestionario nórdico estandarizado para el análisis de síntomas musculoesqueléticos (Kuorinka et al., 1987), en su versión original, traducido al español. El cuestionario se compone de dos partes, un cuestionario general y otra sección para zonas específicas del cuerpo. Solo se validó la primera parte por ser la más utilizada.

\section{Procedimiento}

Como primer paso se realizó la traducción y retro - traducción del instrumento al idioma inglés, para asegurar la fidelidad del contenido a la versión original.

Se realizó el análisis de confiabilidad del instrumento mediante el alfa de Cronbach.

Con el fin de confirmar la validez del instrumento, se realizaron cuatro análisis.

a. Análisis factorial exploratorio, a fin de comprobar la estructura del instrumento.

b. Se utilizó el escalamiento multidimensional no métrico (PROXSCAL), para comprobar la estructura interna del instrumento.

c. Se estimó la confiabilidad test - retest con una sub - muestra de 30 trabajadores, a los cuales se les aplicó el cuestionario una semana después de la primera aplicación. Se evaluó la concordancia entre ambas aplicaciones mediante el estadístico Kappa.

\section{Resultados y discusión}

\section{Análisis de confiabilidad}

El análisis reportó un alfa de Cronbach de o,863 para la escala completa, en la tabla 2 se presentan los valores por ítem, cuando se suprime el elemento. De acuerdo con lo señalado por De Vellis (1991), un valor Alfa de 0,80 a 0,90, indica una confiabilidad muy buena, es decir que el instrumento produce resultados consistentes y coherentes. 
Tabla 2. Resultados del análisis de confiabilidad (alfa de Cronbach).

\begin{tabular}{|l|l|c|}
\hline \multicolumn{1}{|c|}{ Zona corporal } & \multicolumn{1}{|c|}{ Condición } & $\begin{array}{c}\text { Alfa de Cronbach si el } \\
\text { elemento se ha suprimido }\end{array}$ \\
\hline \multirow{4}{*}{ Lumbar (espalda baja) } & 12 meses & 0,855 \\
\cline { 2 - 3 } & Evitar hacer su trabajo & 0,859 \\
\cline { 2 - 3 } & Últimos 7 días & 0,855 \\
\hline \multirow{5}{*}{ Cervical (espalda alta) } & 12 meses & 0,865 \\
\cline { 2 - 3 } & Evitar hacer su trabajo & 0,859 \\
\cline { 2 - 3 } & Últimos 7 días & 0,858 \\
\hline \multirow{5}{*}{ Cadera } & 12 meses & 0,863 \\
\cline { 2 - 3 } & Evitar hacer su trabajo & 0,862 \\
\cline { 2 - 3 } & Últimos 7 días & 0,863 \\
\hline \multirow{5}{*}{ Robillos } & 12 meses & 0,866 \\
\cline { 2 - 3 } & Evitar hacer su trabajo & 0,860 \\
\cline { 2 - 3 } & Últimos 7 días & 0,860 \\
\hline \multirow{5}{*}{ Hodillas } & 12 meses & 0,856 \\
\cline { 2 - 3 } & Evitar hacer su trabajo & 0,858 \\
\cline { 2 - 3 } & Últimos 7 días & 0,857 \\
\hline \multirow{5}{*}{ Codo } & 12 meses & 0,856 \\
\cline { 2 - 3 } & Evitar hacer su trabajo & 0,857 \\
\cline { 2 - 3 } & Últimos 7 días & 0,855 \\
\hline \multirow{2}{*}{ Muñeca } & 12 meses & 0,856 \\
\cline { 2 - 3 } & Evitar hacer su trabajo & 0,858 \\
\cline { 2 - 3 } & Últimos 7 días & 0,858 \\
\hline Cuello & 12 meses & 0,858 \\
\cline { 2 - 3 } & Evitar hacer su trabajo & 0,858 \\
\cline { 2 - 3 } & Últimos 7 días & 0,858 \\
\hline & 12 meses & 0,859 \\
\cline { 2 - 3 } & Evitar hacer su trabajo & 0,860 \\
\cline { 2 - 3 } & Últimos 7 días & 0,859 \\
\hline & & \\
\hline
\end{tabular}

\section{Análisis factorial exploratorio}

Para comprobar la estructura del instrumento, en la versión traducida al español, se realizó el proceso de análisis factorial de tipo exploratorio mediante el método de extracción de máxima verosimilitud, se empleó la rotación varimax con normalización Kaiser. Este análisis se realiza cuando no se tiene certeza de cuál es la estructura del instrumento.

Se obtuvo un valor de bondad de ajuste de $\chi 2=550, p \leq 0,001$, así como una medida de adecuación muestral con un valor $\mathrm{KMO}=0,822$. Ambos estadísticos indican un buen ajuste. Cuanto más cerca está el valor del 1, implica que la relación entre las variables es alta.

A partir de la extracción de factores se obtienen nueve factores, correspondientes a los segmentos corporales considerados en el instrumento, los cuales explican el 72,05\% de la varianza total. Los Eigen value son mayores de uno. Esta estructura factorial es confiable, confirmando lo obtenido en el coeficiente alfa de Cronbach. El único factor que tiene valores muy bajos es el cuello (ver anexo). 


\section{Escalamiento multidimensional PROXSCAL}

Se comprobó la estructura encontrada en las pruebas anteriores, y la cercanía de los ítems, mediante el escalamiento multidimensional. Los resultados mostraron indicadores adecuados del ajuste de la solución (tabla 3). Los estadísticos de stress miden el desajuste de los datos, y son adecuados al acercarse a o, mientras que las medidas de ajuste (la dispersión explicada y el coeficiente de congruencia de Tucker) son adecuadas si se acercan a 1.

Tabla 3. Medidas de ajuste y stress del instrumento.

\begin{tabular}{|l|c|}
\hline Medidas de stress y de ajuste & Valor \\
\hline Estrés bruto normalizado & 0,00103 \\
\hline Estrés-I & $0,03205^{\mathrm{a}}$ \\
\hline Estrés-II & $0,03666^{\mathrm{a}}$ \\
\hline S-Estrés & $0,00188^{\mathrm{b}}$ \\
\hline Dispersión contada para (D,A,F,) & 0,99897 \\
\hline Coeficiente de congruencia de Tucker & 0,99949 \\
\hline PROXSCAL minimiza el estrés bruto normalizado. & \\
\hline a. Factor de escalamiento óptimo $=1,001$. & \\
\hline b. Factor de escalamiento óptimo $=1,000$. & \\
\hline
\end{tabular}

En la figura 3 se muestran las dos coordenadas principales asociadas a los 27 ítems del cuestionario. Se puede apreciar que se agrupan en forma muy clara, lo cual se corresponde con la consistencia interna presentada anteriormente. La única excepción son síntomas en muñecas y hombros en los últimos 12 meses. 
Figura 3. Distribución de los ítems en las coordenadas principales.

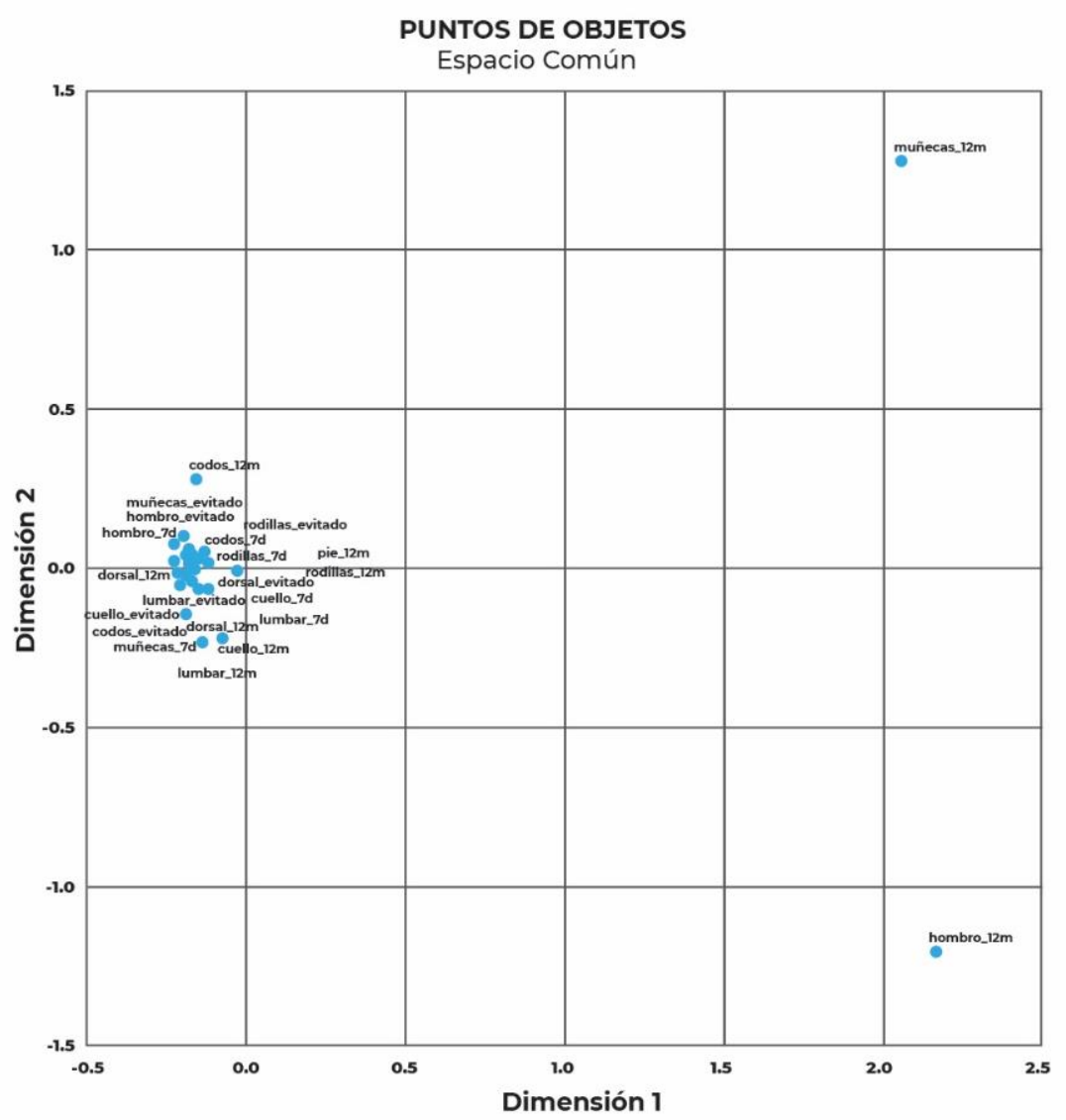

Análisis de la confiabilidad test - retest

Este análisis se realizó para comparar las puntuaciones obtenidas en dos aplicaciones del instrumento, realizadas con 6 días de diferencia. Se compararon las puntuaciones obtenidas en un subgrupo de 27 trabajadores administrativos, seleccionados de entre la muestra anterior.

Se corrió la prueba t para muestras relacionadas a fin de determinar si las medias de las dos aplicaciones del cuestionario nórdico diferían entre sí. La diferencia observada entre las medias resultó ser no significativa estadísticamente $(\mathrm{p}=0,349)$, por lo que se aceptó la hipótesis de la igualdad de las medias de la puntuación en las dos aplicaciones (test y retest).

Se obtuvo el coeficiente de correlación intraclase, como una medida de acuerdo de los valores de las puntuaciones entre los propios casos, el cual mostró una correlación fuerte $(0,625)$ entre las dos mediciones. Esto indica que el instrumento presenta estabilidad temporal, es decir que se obtienen los mismos resultados en diferentes mediciones. 


\section{Discusión}

Los resultados obtenidos en el análisis de la confiabilidad reportaron un coeficiente de alfa entre 0,855 y 0,860, los cuales son mayores a los reportados por Martínez et al. (2014), quienes en una versión española obtuvieron valores entre 0,727 y 0,816. Maradei et al. (2019) reportaron valores muy similares $(\alpha=0,89)$. Esto confirma la confiabilidad del instrumento, como un instrumento para detectar síntomas entre trabajadores, tanto en los últimos 12 meses como a los 7 días.

Los análisis de la validez del instrumento confirmaron la estructura sólida del mismo. Se obtuvo un valor de bondad de ajuste de $\chi^{2}=550, \mathrm{p} \leq 0,001, \mathrm{y}$ un valor $\mathrm{KMO}=0,822$. Los nueve factores obtenidos explican el 72,05\% de la varianza total. Estos resultados contrastan con un estudio realizado en España (Martínez et al., 2014), en el cual se analizó la estructura mediante un análisis factorial, obteniéndose valores que indican un ajuste adecuado (KMO 0,749) y explica el 60,54\% de la varianza. La gran diferencia es que en este estudio se obtuvieron tres factores relacionándolos con la temporalidad de las molestias.

\section{Conclusiones}

Se confirma que el cuestionario nórdico original cumple con el papel de ser un instrumento para tamizaje y detección de trabajadores con molestias, lo cual sirve como base para realizar una evaluación más profunda con ellos.

Se recomienda el uso del instrumento en poblaciones con baja escolaridad, debido a la sencillez de su estructura, lo cual permite la rápida comprensión de su contenido, además de responderse en poco tiempo. 


\section{Referencias}

Bellorín, M., Sirit, Y., Rincón, C., \& Amortegui, M. (2007). Síntomas músculo esqueléticos en trabajadores de una empresa de construcción civil. Salud de los trabajadores, 15(2), 89-98. https://www.redalyc.org/articulo.oa?id=375839287003

De Vellis, R. F. (1991). Scale development. Theory and applications. SAGE Publications. https://doi.org/10.1177/014662169101500413

Instituto Mexicano del Seguro Social (2021). Memoria estadística 2019. http://www,imss,gob,mx/conoce-al-imss/memoria-estadistica-2019

Kuorinka, I., Jonsson, B., Kilbom, A., Vinterberg, H., Biering-Sørensen, F., Andersson, G., \& Jørgensen, K. (1987). Standardised nordic questionnaires for the analysis of musculoskeletal symptoms. Applied ergonomics, 18(3), 233-237. https://doi.org/10.1016/00036870(87)90010-X

Maradei, F., Ardila, C., \& Sanabria, S. (2019). Síntomas musculoesqueléticos en las actividades de cosecha de mora de castilla de Piedecuesta, Colombia. Hacia la Promoción de la Salud, 24(2), 91-106. https://doi.org/10.17151/hpsal.2019.24.2.8

Martínez, M. M., \& Muñoz, R. A. (2017). Validación del cuestionario nórdico estandarizado de síntomas musculoesqueléticos para la población trabajadora chilena, adicionando una escala de dolor. Revista de Salud Pública, 21(2), 43-53. http://repositorio.uchile.cl/bitstream/handle/2250/152284/Validation-of-the-nordicstandardized-questionnaire.pdf? sequence $=1$

Martínez, B., Santo-Domingo, S. S., Bolea, M., Casalod, Y., \& Andres, E. (2014). Validación del cuestionario nórdico musculoesquelético estandarizado en población española, Memorias del ORP2014 - XII Congreso Internacional de Prevención de Riesgos Laborales. http://www,prevencionintegral,com/en/canal-orp/papers/orp-2014/validacioncuestionario-nordico-musculoesqueletico-estandarizado-en-poblacion-espanola

Rojas, L. \& Velarde, M. A. (2020). Síntomas músculos esqueléticos y condiciones laborales en el personal de seguridad, hospital de Lima 2018. Revista Científica de Enfermería, 9(2). Recuperado de https://revista.cep.org.pe/index.php/RECIEN/article/view/29

Secretaría del Trabajo y Prevención Social (2018, 23 de noviembre). NORMA Oficial Mexicana NOM-036-1-STPS-2018. Factores de riesgo ergonómico en el trabajo - identificación, análisis, prevención y control. Parte 1: Manejo manual de cargas. Diario Oficial de la Federación. https://www.dof.gob.mx/nota detalle.php?codigo $=5544579 \& f e c h a=23 / 11 / 2018$

Zamora-Chávez, S, C., Vásquez-Alva, R., Luna-Muñoz, C., \& Carvajal-Villamizar, L. L. (2020). Factores asociados a trastornos musculoesqueléticos en trabajadores de limpieza del servicio de emergencia de un hospital terciario. Revista de la Facultad de Medicina Humana, 2O(3), 388-396. http://dx.doi.org/10.25176/rfmh.v20i3.3055 
Anexo. Valores Eigen, porcentaje de varianza y porcentajes acumulados para los factores del cuestionario.

\begin{tabular}{|c|c|c|c|c|c|c|c|c|c|c|c|}
\hline \multirow{2}{*}{ Zona corporal } & \multirow{2}{*}{ Condición } & \multicolumn{9}{|c|}{ Factor } & \multirow{2}{*}{ Comunalidad } \\
\hline & & $\mathbf{1}$ & $\mathbf{2}$ & 3 & 4 & 5 & 6 & 7 & 8 & 9 & \\
\hline \multirow{3}{*}{$\begin{array}{l}\text { Lumbar (espalda } \\
\text { baja) }\end{array}$} & 12 meses & 0,722 & & & & & & & & & 0,599 \\
\hline & Evitar hacer su trabajo & 0,609 & & & & & & & & & 0,718 \\
\hline & Últimos 7 días & 0,635 & & & & & & & & & 0,568 \\
\hline \multirow{3}{*}{$\begin{array}{l}\text { Cervical } \\
\text { (espalda alta) }\end{array}$} & 12 meses & & 0,825 & & & & & & & & 0,763 \\
\hline & Evitar hacer su trabajo & & 0,563 & & & & & & & & 0,603 \\
\hline & Últimos 7 días & & 0,666 & & & & & & & & 0,590 \\
\hline \multirow{3}{*}{ Cadera } & 12 meses & & & 0,718 & & & & & & & 0,690 \\
\hline & Evitar hacer su trabajo & & & 0,608 & & & & & & & 0,643 \\
\hline & Últimos 7 días & & & 0,808 & & & & & & & 0,757 \\
\hline \multirow{3}{*}{ Tobillos } & 12 meses & & & & 0,794 & & & & & & 0,710 \\
\hline & Evitar hacer su trabajo & & & & 0,636 & & & & & & 0,576 \\
\hline & Últimos 7 días & & & & 0,766 & & & & & & 0,684 \\
\hline \multirow[t]{3}{*}{ Rodillas } & 12 meses & & & & & 0,826 & & & & & 0,753 \\
\hline & Evitar hacer su trabajo & & & & & 0,601 & & & & & 0,623 \\
\hline & Últimos 7 días & & & & & 0,723 & & & & & 0,631 \\
\hline \multirow{3}{*}{ Hombro } & 12 meses & & & & & & 0,502 & & & & 0,434 \\
\hline & Evitar hacer su trabajo & & & & & & 0,632 & & & & 0,518 \\
\hline & Últimos 7 días & & & & & & 0,798 & & & & 0,703 \\
\hline \multirow[t]{3}{*}{ Codo } & 12 meses & & & & & & & 0,828 & & & 0,719 \\
\hline & Evitar hacer su trabajo & & & & & & & 0,567 & & & 0,412 \\
\hline & Últimos 7 días & & & & & & & 0,672 & & & 0,488 \\
\hline \multirow{3}{*}{ Muñeca } & 12 meses & & & & & & & & 0,708 & & 0,620 \\
\hline & Evitar hacer su trabajo & & & & & & & & 0,581 & & 0,471 \\
\hline & Últimos 7 días & & & & & & & & 0,664 & & 0,495 \\
\hline \multirow{6}{*}{ Cuello } & 12 meses & & & & & & & & & 0,488 & 0,442 \\
\hline & Evitar hacer su trabajo & & & & & & & & & 0,181 & 0,313 \\
\hline & Últimos 7 días & & & & & & & & & 0,383 & 0,431 \\
\hline & Eigen value & 6,964 & 2,334 & 1,792 & 1,71 & 1,544 & 1,439 & 1,371 & 1,2 & 1,1 & \\
\hline & \% de varianza & 25,79 & 8,64 & 6,64 & 6,33 & 5,72 & 5,33 & 5,08 & 4,44 & 4,07 & \\
\hline & \% acumulado & 25,79 & 34,44 & 41,07 & 47,41 & 53,12 & 58,46 & 63.53 & 67,98 & 72,05 & \\
\hline
\end{tabular}

